\title{
Phylogenetic Analysis of Butyrivibrio Strains Reveals Three Distinct Groups of Species within the Clostridium Subphylum of the Gram-Positive Bacteria
}

\author{
ANNE WILLEMS, ${ }^{*}$ MONICA AMAT-MARCO, AND MATTHEW D. COLLINS \\ Department of Microbial Physiology, Institute of Food Research, Reading Laboratory, \\ Reading RG6 6BZ, United Kingdom
}

\begin{abstract}
The phylogenetic positions of $\mathbf{4 0}$ Butyrivibrio strains were determined by performing a comparative sequence analysis of the 16S rRNA genes of these organisms. We found that all of the strains which we studied belong to cluster XIVa (M. D. Collins, P. A. Lawson, A. Willems, J. J. Cordoba, J. Fernandez-Garayzabal, P. Garcia, J. Cai, H. Hippe, and J. A. E. Farrow, Int. J. Syst. Bacteriol. 44:812-826, 1994) of the Clostridium subphylum of the gram-positive bacteria, which also includes several Clostridium, Coprococcus, Eubacterium, and Ruminococcus species. We also found that the Butyrivibrio strains which we examined were genotypically heterogeneous and exhibited 12 distinct rRNA sequence types. The 12 rRNA sequence types formed three distinct lineages in cluster XIVa, which were separate from each other and from all other species belonging to this cluster. One lineage consisted of strains which exhibited a single rRNA type and corresponded to the species Butyrivibrio crossotus. The second lineage consisted of 12 strains designated Butyrivibrio fibrisolvens which exhibited seven distinct rRNA sequence types. The type strain of $B$. fibrisolvens was a member of this lineage, but its position was peripheral. The third lineage comprised 26 B. fibrisolvens strains which exhibited four distinct rRNA sequence types. Tree topology and sequence divergence considerations indicated that the three lineages correspond to three separate genera and that the genus Butyrivibrio should be restricted to the group that contains the type strain of $B$. fibrisolvens.
\end{abstract}

The genus Butyrivibrio as currently defined (1) contains curved, non-spore-forming, strictly anaerobic, rod-shaped bacteria that are motile by means of one or more polar to subpolar flagella. Butyrivibrio strains are gram negative, but some strains (including the type strain) have been shown to possess an atypical gram-positive cell wall ultrastructure $(4,7,12)$. These organisms have a fermentative metabolism, use mainly carbohydrates, and characteristically produce butyrate from the fermentation of glucose or mannose $(1,11)$. The butyrivibrios are a major component of the ruminal microflora and have been isolated from the gastrointestinal tracts of various animals, as well as from human, rabbit, and horse feces $(1,11)$. They contribute to fiber digestion in both animals $(11,18,21)$ and humans (24) through degradation of cellulose and xylan (10, $18,19,27)$. The following two Butyrivibrio species are currently recognized: Butyrivibrio fibrisolvens (3), the type species of the genus, which includes monotrichous isolates obtained from animals, and Butyrivibrio crossotus (20), which includes lophotrichous isolates obtained from human feces. Because of the considerable morphological $(3,7)$, metabolic $(2,23,25)$, and serological $(9,17)$ diversity displayed by Butyrivibrio strains, it is generally accepted that this genus contains several more species $(1,3,11,25)$. Early attempts to subdivide the genus on the basis of the phenotypic criteria mentioned above were not very successful because of the extreme heterogeneity of the strains $(9,25)$. More recently, large numbers of Butyrivibrio strains have been studied by chromosomal DNA-DNA hybridization and extracellular polysaccharide analyses. The results of these analyses also revealed the substantial heterogeneity in the genus and identified several groups of strains which may represent new species $(15,16,26)$. The phylogenetic diversity

* Corresponding author. Mailing address: Department of Microbial Physiology, Institute of Food Research, Reading Laboratory, Earley Gate, Whiteknights Road, Reading RG6 6BZ, United Kingdom. of the various groups identified by DNA-DNA hybridization is not clear at this time. It is not known whether these genotypically distinct organisms form a monophyletic group or constitute phenotypically similar but phylogenetically remotely related groups of species, as is the case, for example, with the genus Ruminococcus (22). To investigate the phylogenetic diversity of the genus Butyrivibrio, we compared partial $16 \mathrm{~S}$ rRNA gene sequences of 40 Butyrivibrio strains. Our results indicate that the genus Butyrivibrio, as presently defined, is phylogenetically heterogeneous and encompasses three remotely related species groups.

\section{MATERIALS AND METHODS}

Strains used and determination of $16 \mathrm{~S}$ rRNA gene sequences. The Butyrivibrio strains which we used are listed in Table 1 . Lyophilized cells were resuspended in $500 \mu \mathrm{l}$ of TES buffer (0.05 M Tris-HCl, $0.005 \mathrm{M}$ EDTA, $0.05 \mathrm{M} \mathrm{NaCl} ; \mathrm{pH} 8.0)$, and total DNA was extracted by the method of Lawson et al. (14). The almost complete 16S rRNA gene was amplified by PCR by using conserved primers close to the $3^{\prime}$ and $5^{\prime}$ ends of the gene. The amplified fragment corresponded to positions 30 to 1521 of the Escherichia coli $16 \mathrm{~S}$ rRNA gene. The PCR products were purified by using a Prep-A-Gene kit (Bio-Rad, Hercules, Calif.) according to the manufacturer's instructions and were sequenced directly by using a Taq Dye-Deoxy terminator cycle sequencing kit (Applied Biosystems, Inc., Foster City, Calif.) and a model 373A automatic DNA sequencer (Applied Biosystems, Inc.).

Phylogenetic analysis of the sequence data. Phylogenetic analyses were performed by using the Genetics Computer Group package (6) and the PHYLIP phylogeny inference package (8). To determine the closest known relatives of the Butyrivibrio strains, we performed preliminary searches in the EMBL data library with the program FASTA (6). The resulting sequences and the sequences of other strains known to belong to the same phylogenetic group were retrieved from the EMBL, GenBank, and Ribosomal Database Project data libraries and aligned with the sequences of the Butyrivibrio strains by using the program PILEUP (6). The rRNA alignment was corrected manually, and approximately 100 bases at the $5^{\prime}$ end of the molecule were omitted from further analyses because of alignment uncertainties. A distance matrix was obtained by using the programs PRETTY (6) and DNADIST (8) and the Kimura-2 parameter, and a phylogenetic tree based on the neighbor-joining method was constructed by using the program NEIGHBOR (8). The stability of the groups was determined by performing a bootstrap analysis ( 500 replications) by using the programs DNABOOT, DNADIST, NEIGHBOR, and CONSENSE (8). For comparison, 
TABLE 1. Strains used

\begin{tabular}{|c|c|c|c|}
\hline Strain $^{a}$ & Other designation(s) & Source $^{b}$ & Origin \\
\hline \multicolumn{4}{|l|}{ B. fibrisolvens } \\
\hline rRNA type 1 strain NCDO $2221^{\mathrm{Tc}}$ & D1, ATCC 19171 & Bryant & Bovine rumen \\
\hline \multicolumn{4}{|l|}{ rRNA type 2 strains } \\
\hline $\mathrm{NCDO} 2222^{c}$ & A38, ATCC 27208 & Bryant & Bovine rumen \\
\hline Bu 25 & K202-21-07-6D & van Gylswyk & \\
\hline \multicolumn{4}{|l|}{ rRNA type 3 strains } \\
\hline NCDO $2398^{c}$ & $\mathrm{~B} 835$ & Mann & Heifer rumen \\
\hline NCDO 2417 & B834 & Hobson & \\
\hline $\mathrm{Bu} 22$ & $\mathrm{~K} 27 \mathrm{BF} 1,1$ & van Gylswyk & \\
\hline rRNA type 4 strain NCDO $2432^{c}$ & Ce65 & van Gylswyk & Sheep rumen \\
\hline rRNA type 5 strain NCDO $2434^{c}$ & $\mathrm{Bu} 42, \mathrm{~K} 13-26-037-\mathrm{A}$ & van Gylswyk & \\
\hline \multicolumn{4}{|l|}{ rRNA type 6 strains } \\
\hline NCDO $2435^{c}$ & Bu 24, K10-02-04-6B & van Gylswyk & \\
\hline NCDO 2436 & $\mathrm{Bu} 44, \mathrm{Ce} 78$ & van Gylswyk & Sheep rumen \\
\hline Bu 36 & $\mathrm{Ce} 47$ & van Gylswyk & Sheep rumen \\
\hline rRNA type 7 strain $\mathrm{Bu} 43^{c}$ & Ce64 & van Gylswyk & Sheep rumen \\
\hline \multicolumn{4}{|l|}{ rRNA type 8 strains } \\
\hline $\mathrm{NCDO} 2223^{c}$ & $\mathrm{Bu} 49$ & Bryant & Bovine rumen \\
\hline NCDO 2418 & $\mathrm{Bu} 27,19$ & van Gylswyk & Sheep rumen \\
\hline NCDO 2419 & Bu 31, CXS 18 & van Gylswyk & Sheep rumen \\
\hline NCDO 2421 & $\mathrm{Bu} 41, \mathrm{Ce} 58$ & van Gylswyk & Sheep rumen \\
\hline Bu 12 & $\mathrm{~K} 29 \mathrm{CF}, 12$ & van Gylswyk & Sheep rumen \\
\hline $\mathrm{Bu} 15$ & $\mathrm{~K} 29 \mathrm{HF}, 15$ & van Gylswyk & Sheep rumen \\
\hline Bu 17 & $\mathrm{~K} 13 \mathrm{KF}, 17$ & van Gylswyk & Sheep rumen \\
\hline Bu 26 & 18 & van Gylswyk & Sheep rumen \\
\hline Bu 28 & 20 & van Gylswyk & Sheep rumen \\
\hline Bu 29 & 21 & van Gylswyk & Sheep rumen \\
\hline Bu 30 & 24 & van Gylswyk & Sheep rumen \\
\hline $\mathrm{Bu} 32$ & CXS13 & van Gylswyk & Sheep rumen \\
\hline $\mathrm{Bu} 33$ & 22 & van Gylswyk & Sheep rumen \\
\hline Bu 34 & $\mathrm{Ce} 36$ & van Gylswyk & Sheep rumen \\
\hline Bu 35 & $\mathrm{Ce} 46$ & van Gylswyk & Sheep rumen \\
\hline $\mathrm{Bu} 37$ & $\mathrm{Ce} 51$ & van Gylswyk & Sheep rumen \\
\hline $\mathrm{Bu} 38$ & $\mathrm{Ce} 52$ & van Gylswyk & Sheep rumen \\
\hline Bu 39 & $\mathrm{Ce} 53$ & van Gylswyk & Sheep rumen \\
\hline $\mathrm{Bu} 40$ & Ce56 & van Gylswyk & Sheep rumen \\
\hline $\mathrm{Ce} 52$ & Bu 38 & van Gylswyk & Sheep rumen \\
\hline \multicolumn{4}{|l|}{ rRNA type 9 strains } \\
\hline NCDO $2249^{c}$ & Nor37 & NIRD & Bovine rumen \\
\hline NCDO 2400 & 1L 6-31 & NIRD & Bovine rumen \\
\hline NCDO 2438 & $\mathrm{Ce} 74$ & van Gylswyk & Sheep rumen \\
\hline rRNA type 10 strain NCDO $2397^{\circ}$ & $\mathrm{JL}$ & Leatherwood & \\
\hline \multicolumn{4}{|l|}{ rRNA type 11 strains } \\
\hline NCDO $2399^{\circ}$ & Bu 21, RO3-21-08-7A & van Gylswyk & \\
\hline $\mathrm{Bu} 21$ & NCDO 2399, RO3-21-08-7A & van Gylswyk & \\
\hline \multicolumn{4}{|l|}{ B. crossotus rRNA type 12 strains } \\
\hline NCDO $2416^{\mathrm{T} c}$ & $\mathrm{Bu} 46, \mathrm{~T} 940 \mathrm{~A}$ & VPI & Human feces \\
\hline NCDO 2415 & $\mathrm{Bu} 45, \mathrm{C} 3824$ & VPI & Human feces \\
\hline
\end{tabular}

${ }^{a}$ NCDO, National Collection of Dairy Organisms, Reading, United Kingdom.

${ }^{b}$ Bryant, M. P. Bryant, University of Illinois, Urbana; Hobson, P. N. Hobson, Rowett Research Institute, Aberdeen, United Kingdom; Leatherwood, J. M. Leatherwood, North Carolina State University, Raleigh; Mann, S. O. Mann, Rowett Research Institute, Aberdeen, United Kingdom; van Gylswyk, N. O. van Gylswyk, Veterinary Research Institute, Onderstepoort, South Africa; NIRD, National Institute for Research in Dairying, Shinfield, United Kingdom; VPI, Virginia Polytechnic Institute and State University, Blacksburg.

c Strain whose nearly complete $16 \mathrm{~S}$ rRNA gene sequence was determined as a representative of the rRNA type. Partial 16S rRNA gene sequences (approximately 600 bases proximal to the $5^{\prime}$ end of the rRNA, including diagnostic variable regions V1 to V3) were determined for the other strains. The sequences determined for all members of each rRNA type were identical.

we also constructed a pairwise similarity matrix by repeatedly using the program GAP (6). The similarity values were converted to nucleotide substitution $\left(K_{\text {nuc }}\right)$ values (13), and a phylogenetic tree was drawn with the program NEIGHBOR.

Nucleotide sequence accession numbers. The $16 \mathrm{~S}$ rRNA gene sequences of $B$. fibrisolvens $\mathrm{NCDO} 2221^{\mathrm{T}}$ ( $\mathrm{T}=$ type strain), NCDO 2222, NCDO 2398, NCDO 2432 , NCDO 2434, NCDO 2435, Bu 43, NCDO 2223, NCDO 2249, NCDO 2397 , and NCDO 2399 and B. crossotus NCFB $2416^{\mathrm{T}}$ have been deposited in the EMBL data library under accession numbers X89970 to X89981.

\section{RESULTS AND DISCUSSION}

To shed light on the phylogenetic diversity of the genus Butyrivibrio, we compared the partial 16S rRNA gene se- quences of 40 Butyrivibrio strains. Our initial sequence characterization and comparison of a stretch of approximately 600 to 700 nucleotides encoding the $5^{\prime}$ end of the molecule revealed that there were 12 rRNA types or groups among the strains which we studied (Table 1). Consequently, the complete 16S rRNA gene sequence of one representative strain of each group was determined. The derived $16 \mathrm{~S}$ rRNA primary sequences consisted of between 1,478 and 1,496 nucleotides and represented approximately $97 \%$ of the rRNA molecule. One of the 12 rRNA types which we found consisted of strains of $B$. 


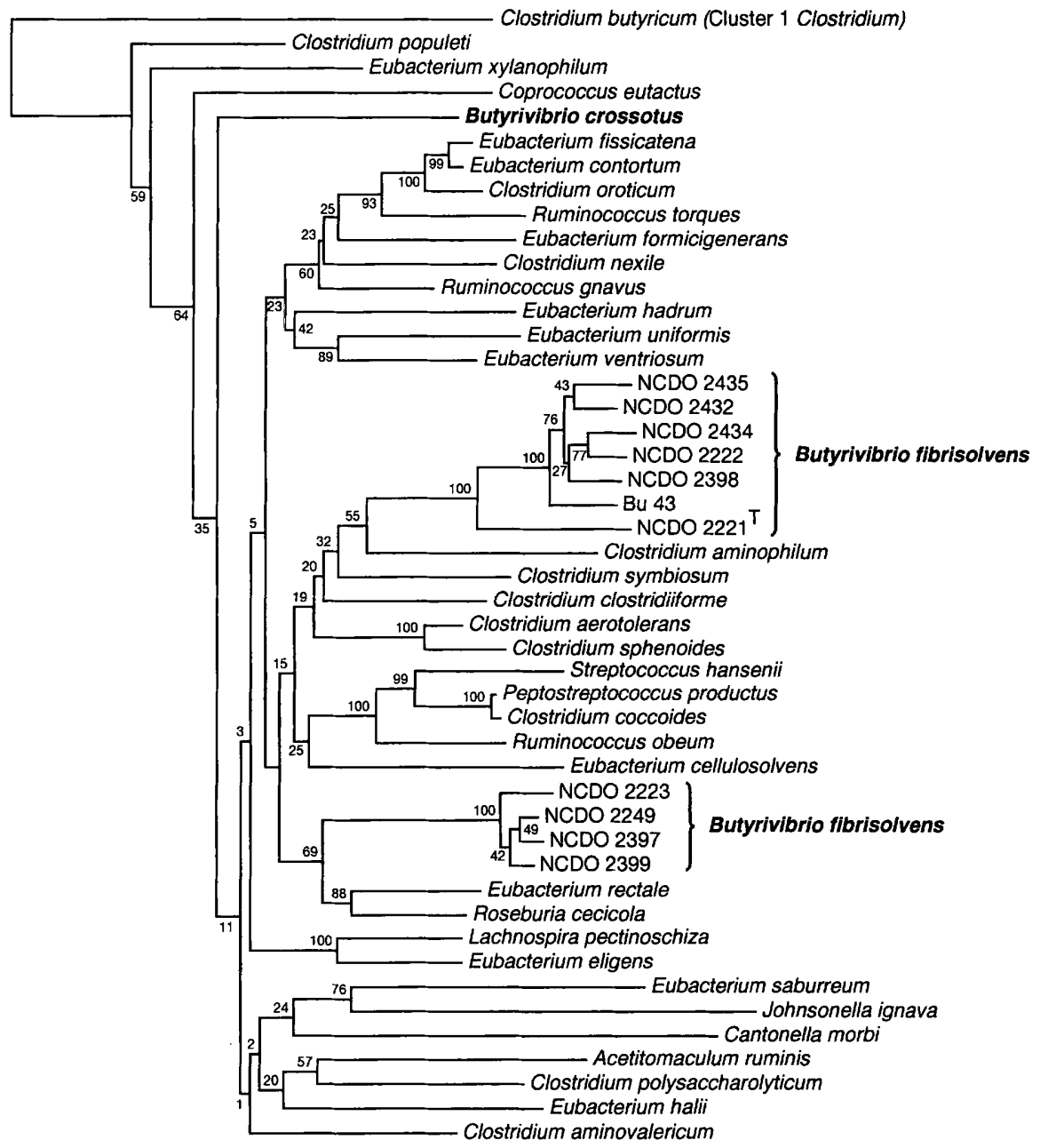

FIG. 1. Unrooted phylogenetic tree obtained by performing a neighbor-joining analysis of a distance matrix obtained from a multiple-sequence alignment, showing the positions of Butyrivibrio strains within cluster XIVa (5) of the Clostridium subphylum of the gram-positive bacteria. Bootstrap values (expressed as percentages of 500 replications) are shown at the branch points; values of $90 \%$ or more were considered significant.

crossotus, while the remaining 11 rRNA types included only strains designated $B$. fibrisolvens. rRNA type 8 was the largest group, consisting of 20 of the 40 strains studied. rRNA types 3 , 6 , and 9 each contained three strains; rRNA type 2 contained two strains; and rRNA types $1,4,5,7,10$, and 11 were each represented by a single strain. rRNA type 1 contained the type strain of $B$. fibrisolvens. The levels of sequence similarity for strains belonging to the different Butyrivibrio rRNA types are shown in Table 2. Although there is no precise or absolute level of sequence similarity for delineating species, the levels of sequence divergence found for representatives of the various Butyrivibrio rRNA groups indicate that these taxa are at least separate species. This view is supported by the results of studies in which extracellular polysaccharide analysis and DNADNA hybridization were used to classify Butyrivibrio strains $(15,16,26)$. These results confirmed the separateness of strains D1 (corresponding to rRNA type 1) and A38 (rRNA type 2) and placed strains $\mathrm{Bu} 49, \mathrm{Ce} 51, \mathrm{Ce} 52$, and 12 in the same DNA group (rRNA type 8 ). However, one difference between the results of the DNA-DNA hybridization study of Mannarelli (15) and the results of our investigation was apparent. Mannarelli (15) reported that strains 1L6-31, Nor37, and B835 belong to a single DNA homology group. We found that while strains 1L6-31 and Nor37 constitute a distinct rRNA group (rRNA type 9), strain B835 belonged to an rRNA subline that was quite separate (rRNA type 3 ). The level of $16 \mathrm{~S}$ rRNA sequence divergence between the strains belonging to these rRNA types (approximately 13\%) is clearly incompatible with the hypothesis that these organisms belong to the same species.

Comparative sequence searches of the EMBL and GenBank data libraries revealed that all of the Butyrivibrio strains which we examined were most closely related to species belonging to cluster XIVa of the Clostridium subphylum of the gram-positive bacteria as defined by Collins et al. (5). To determine the phylogenetic positions of the Butyrivibrio rRNA types within cluster XIVa, we performed a comprehensive phylogenetic analysis in which we included all known members of cluster XIVa and Clostridium butyricum (the type species of the genus Clostridium) as an outgroup. The phylogenetic tree that resulted from our analysis based on multiple sequence alignments is shown in Fig. 1. This tree was very similar to the tree obtained after pairwise alignment of the sequences (data not shown) in that all significant groups were present on both trees. The treeing analyses revealed that the 12 Butyrivibrio rRNA types formed three separate lineages within cluster XIVa (Fig. 1 ). The $B$. crossotus (rRNA type 12) strains formed a distinct 
TABLE 2. Similarity values for the $16 \mathrm{~S}$ rRNA genes of representative strains of the different Butyrivibrio rRNA types

\begin{tabular}{|c|c|c|c|c|c|c|c|c|c|c|c|}
\hline \multirow[b]{2}{*}{ Strain } & \multicolumn{11}{|c|}{$\%$ Similarity with: } \\
\hline & 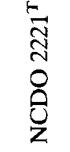 & $\begin{array}{l}\text { Ñ } \\
\text { N } \\
0 \\
0 \\
\mathbf{Z}\end{array}$ & 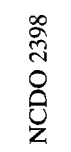 & 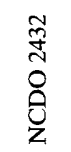 & 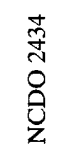 & 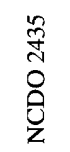 & $\stackrel{?}{\overrightarrow{7}}$ & 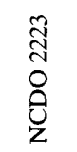 & 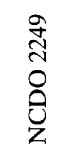 & 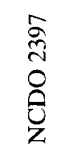 & 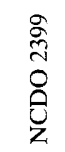 \\
\hline $\begin{array}{l}\text { B. fibrisolvens NCDO } 2222 \\
\text { (rRNA type 2) }\end{array}$ & 94.1 & & & & & & & & & & \\
\hline $\begin{array}{l}\text { B. fibrisolvens NCDO } 2398 \\
\text { (rRNA type } 3 \text { ) }\end{array}$ & 93.6 & 97.1 & & & & & & & & & \\
\hline $\begin{array}{l}\text { B. fibrisolvens NCDO } 2432 \\
\text { (rRNA type } 4 \text { ) }\end{array}$ & 93.6 & 96.6 & 97.1 & & & & & & & & \\
\hline $\begin{array}{l}\text { B. fibrisolvens NCDO } 2434 \\
\text { (rRNA type 5) }\end{array}$ & 93.6 & 98.5 & 97.6 & 96.2 & & & & & & & \\
\hline $\begin{array}{l}\text { B. fibrisolvens NCDO } 2435 \\
\text { (rRNA type } 6 \text { ) }\end{array}$ & 93.1 & 96.8 & 97.0 & 97.3 & 97.1 & & & & & & \\
\hline $\begin{array}{l}\text { B. fibrisolvens Bu } 43 \\
\text { (rRNA type } 7 \text { ) }\end{array}$ & 93.1 & 95.9 & 96.5 & 97.2 & 96.8 & 96.8 & & & & & \\
\hline $\begin{array}{l}\text { B. fibrisolvens NCDO } 2223 \\
\text { (rRNA type } 8 \text { ) }\end{array}$ & 88.2 & 86.9 & 86.9 & 87.1 & 87.1 & 87.0 & 86.6 & & & & \\
\hline $\begin{array}{l}\text { B. fibrisolvens NCDO } 2249 \\
\text { (rRNA type 9) }\end{array}$ & 88.2 & 86.8 & 86.8 & 87.2 & 87.3 & 87.1 & 86.8 & 98.0 & & & \\
\hline $\begin{array}{l}\text { B. fibrisolvens NCDO } 2397 \\
\text { (rRNA type 10) }\end{array}$ & 88.3 & 87.0 & 87.1 & 87.4 & 87.4 & 87.3 & 87.1 & 98.4 & 99.0 & & \\
\hline $\begin{array}{l}\text { B. fibrisolvens NCDO } 2399 \\
\text { (rRNA type 11) }\end{array}$ & 88.0 & 86.7 & 86.8 & 87.1 & 87.2 & 87.2 & 86.9 & 97.7 & 98.7 & 98.8 & \\
\hline $\begin{array}{l}\text { B. crossotus NCDO } 2416^{\mathrm{T}} \\
\text { (rRNA type } 12 \text { ) }\end{array}$ & 89.2 & 88.4 & 88.3 & 88.1 & 87.9 & 88.1 & 87.8 & 90.8 & 90.3 & 90.5 & 90.3 \\
\hline
\end{tabular}

lineage that was only remotely related to the other Butyrivibrio rRNA types (levels of sequence similarity, 87.8 to $90.8 \%$ ) (Table 2 ) and all members of cluster XIVa (levels of sequence similarity, 86.2 to $91.4 \%$ ). The $B$. fibrisolvens rRNA type 1 to 7 strains formed a second lineage within cluster XIVa and exhibited levels of sequence similarity of 93.1 to $98.5 \%$ with each other (Table 2), but significantly lower levels of sequence similarity $(85.5$ to $92.1 \%)$ with other members of cluster XIVa. The rRNA type 1 strain, which is the type strain of the type species of the genus Butyrivibrio, was the most peripheral member of this lineage and exhibited levels of sequence similarity of 93.1 to $94.1 \%$ with rRNA type 2 to 7 strains (Table 2). The rRNA type 2 to 7 strains were more closely related to each other and exhibited intragroup levels of sequence similarity of 95.9 to $98.5 \%$ (Table 2). The third Butyrivibrio lineage was made up of rRNA type 8 to 11 strains, which exhibited levels of sequence similarity of 97.7 to $99.0 \%$ with each other (Table 2) and significantly lower levels of relatedness (levels of sequence similarity, 84.3 to $93.5 \%$ ) with members of other species belonging to cluster XIVa. The three major phylogenetic lineages identified by the comparative rRNA gene sequence analysis were not consistent with the previous phenotypic groups based on cellular morphology and metabolism. For example, the two metabolic groups defined by Shane et al. (25) in the genus Butyrivibrio did not correlate with the two major phylogenetic groups (Table 2) within the species; group 1 of Shane et al. included nine rRNA type 8 strains and one rRNA type 6 strain (strain Ce47), while group 2 of Shane et al. contained rRNA type 4 strain Ce65, rRNA type 6 strain Ce78, rRNA type 7 strain Ce64, and rRNA type 9 strain Ce74. On the basis of cell wall structure Dibbayawan et al. (7) placed $B$. fibrisolvens $\mathrm{B} 835, \mathrm{Bu} 49$, and Nor37 in a single group that was separate from $B$. fibrisolvens D1. Phylogenetically, these four strains belong to four different rRNA types, with B835 belonging to the same major lineage as the type strain of $B$. fibrisol- vens and $\mathrm{Bu} 49$ and Nor37 belonging to a different lineage (Table 2).

From the genotypic data presented here and elsewhere (16), it is clear that the different $B$. fibrisolvens rRNA types correspond to separate species. Furthermore, 16S rRNA sequence divergence values and tree topology considerations (supported by bootstrap data [Fig. 1]) indicate that $B$. fibrisolvens rRNA types 1 to $7, B$. fibrisolvens rRNA types 8 to 11 , and $B$. crossotus constitute three remotely related lineages within Clostridium cluster XIVa, each meriting status as a separate genus. The numbers of species in these lineages are likely to increase, and/or other major lineages may be identified as additional Butyrivibrio strains, including members of other genotypically distinct groups described by Mannarelli et al. (16), are included in future phylogenetic studies. However, phenotypic data that support division of the genus Butyrivibrio along phylogenetic lines are not available at this time and may not be readily forthcoming. Most Butyrivibrio strains closely resemble one another; for example, they ferment similar carbohydrates, produce the same fermentation products, and share many other traits, including cell wall structure. The emerging picture of a phylogenetically dispersed genus Butyrivibrio also raises serious questions concerning the taxonomy of the other members of Clostridium cluster XIVa. At present this group encompasses a broad spectrum of species belonging to various genera (viz., the genera "Acetitomaculum," Butyrivibrio, Clostridium, Coprococcus, Eubacterium, Roseburia, and Ruminococcus) having diverse phenotypes (e.g., cocci and rods, spore forming and non-spore forming). The phylogenetic intermixing of many of these taxa and the general complexity of the branching within cluster XIVa (Fig. 1) indicate that it may be difficult to divide this group into genera that are phenotypically coherent and distinguishable. It is clear that several major lines within cluster XIVa are currently represented by a single species or strain. As PCR-based rRNA sequence analysis is routinely used to 
phylogenetically characterize anaerobic isolates and study diversity, it may be shown that many of these isolated lines represent the nuclei of new genera, as is the case with butyrivibrios. The problem of phenotypic circumscription and differentiation which is currently preventing comprehensive taxonomic reorganization in this and other clostridial clusters may then prove to be even more complex.

\section{ACKNOWLEDGMENT}

This work was supported in part by grants CT93-0119 and CT930194 from the Commission of the European Communities.

\section{REFERENCES}

1. Bryant, M. P. 1984. Genus IV. Butyrivibrio Bryant and Small 1956, 18, emend. Moore, Johnson and Holdeman 1976, p. 641-643. In N. R. Krieg and J. G. Holt (ed.), Bergey's manual of systematic bacteriology, vol. 1. The Williams and Wilkins Co., Baltimore.

2. Bryant, M. P., and I. M. Robinson. 1962. Some nutritional characteristics of predominant culturable ruminal bacteria. J. Bacteriol. 84:605-614.

3. Bryant, M. P., and N. Small. 1956. The anaerobic monotrichous butyric acid-producing curved rod-shaped bacteria of the rumen. J. Bacteriol. 72: $16-21$.

4. Cheng, K.-J., and J. W. Costerton. 1977. Ultrastructure of Butyrivibrio fibrisolvens: a gram-positive bacterium? J. Bacteriol. 129:1506-1512.

5. Collins, M. D., P. A. Lawson, A. Willems, J. J. Cordoba, J. FernandezGarayzabal, P. Garcia, J. Cai, H. Hippe, and J. A. E. Farrow. 1994. The phylogeny of the genus Clostridium: proposal of five new genera and eleven new species combinations. Int. J. Syst. Bacteriol. 44:812-826.

6. Devereux, J., P. Haeberli, and D. Smithies. 1984. A comprehensive set of sequence analysis programs for the VAX. Nucleic Acids Res. 12:387-395.

7. Dibbayawan, T., G. Cox, K. Y. Cho, and D. M. Dwarte. 1985. Cell wall and plasma membrane architecture of Butyrivibrio spp. J. Ultrastruct. Res. 90: 286-293.

8. Felsenstein, J. 1989. PHYLIP-phylogeny inference package (version 3.2). Cladistics 5:164-166.

9. Hazlewood, G. P., M. K. Theodorou, A. Hutchings, D. J. Jordan, and G. Galfre. 1986. Preparation and characterization of monoclonal antibodies to a Butyrivibrio sp. and their potential use in the identification of rumen butyrivibrios, using an enzyme-linked immunosorbent assay. J. Gen. Microbiol. 132:43-52.

10. Hespell, R. B. 1992 . Fermentation of xylans by Butyrivibrio fibrisolvens and Thermoanaerobacter strain B6A: utilization of uronic acids and xylanolytic activities. Curr. Microbiol. 25:189-195.

11. Hespell, R. B. 1992. The genera Butyrivibrio, Lachnospira, and Roseburia, p. 2022-2033. In A. Balows, H. G. Trüper, M. Dworkin, W. Harder, and K.-H.
Schleifer (ed.), The prokaryotes. A handbook on the biology of bacteria: ecophysiology, isolation, identification, application, 2nd ed., vol. II. SpringerVerlag, New York.

12. HespelI, R. B., K. Kato, and J. W. Costerton. 1993. Characterization of the cell wall of Butyrivibrio species. Can. J. Microbiol. 39:912-921.

13. Hori, H., and S. Osawa. 1979. Evolutionary change in 5S rRNA secondary structure and phylogenetic tree of 54 SS rRNA species. Proc. Natl. Acad. Sci. USA 76:381-385.

14. Lawson, P. A., S. E. Gharbia, H. N. Shah, and D. R. Clark. 1989. Recognition of Fusobacterium nucleatum subgroups Fn-1, Fn-2 and Fn-3 by ribosomal RNA gene restriction patterns. FEMS Microbiol. Lett. 65:41-46.

15. Mannarelli, B. M. 1988. Deoxyribonucleic acid relatedness among strains of the species Butyrivibrio fibrisolvens. Int. J. Syst. Bacteriol. 38:340-347.

16. Mannarelli, B. M., R. J. Stack, D. Lee, and L. Ericsson. 1990. Taxonomic relatedness of Butyrivibrio, Lachnospira, Roseburia, and Eubacterium species as determined by DNA hybridization and extracellular-polysaccharide analysis. Int. J. Syst. Bacteriol. 40:370-378.

17. Margherita, S. S., and R. E. Hungate. 1963. Serological analysis of Butyrivibrio from the bovine rumen. J. Bacteriol. 86:855-860.

18. Miron, J. 1991. The hydrolysis of lucerne cell-wall monosaccharide components by monocultures or pair combinations of defined ruminal bacteria. J. Appl. Bacteriol. 70:245-252.

19. Miron, J., and D. Benghedahlia. 1993. Digestion of cell-wall monosaccharides of ryegrass and alfalfa hays by the ruminal bacteria Fibrobacter succinogenes and Butyrivibrio fibrisolvens. Can. J. Microbiol. 39:780-786.

20. Moore, W. E. C., J. L. Johnson, and L. V. Holdeman. 1976. Emendation of Bacteroidaceae and Butyrivibrio and description of Desulfomonas gen. nov. and ten new species in the genera Desulfomonas, Butyrivibrio, Eubacterium, Clostridium, and Ruminococcus. Int. J. Syst. Bacteriol. 26:238-252.

21. Orpin, C. G., S. D. Mathiesen, Y. Greenwood, and A. S. Blix. 1985. Seasonai changes in the ruminal microflora of the high-arctic Svalbard reindeer (Rangifer tarandus platyrhynchus). Appl. Environ. Microbiol. 50:144-151.

22. Rainey, F. A., and P. H. Janssen. 1995. Phylogenetic analysis by $16 \mathrm{~S}$ ribosomal DNA sequence comparison reveals two unrelated groups of species within the genus Ruminococcus. FEMS Microbiol. Lett. 129:69-74.

23. Roché, C., H. Albertyn, N. O. van Gylswyk, and A. Kistner. 1973. The growth response of cellulolytic acetate-utilizing and acetate-producing butyrivibrios to volatile fatty acids and other nutrients. J. Gen. Microbiol. 78:253-260.

24. Rumney, C. J., S. H. Duncan, C. Henderson, and C. S. Stewart. 1995. Isolation and characteristics of a wheatbran-degrading Butyrivibrio from human feces. Lett. Appl. Microbiol. 20:232-236.

25. Shane, B. S., L. Gouws, and A. Kistner. 1969. Cellulolytic bacteria occurring in the rumen of sheep conditioned to low-protein teff hay. J. Gen. Microbiol. 55:445-457.

26. Stack, R. J. 1988. Neutral sugar composition of extracellular polysaccharides produced by strains of Butyrivibrio fibrisolvens. Appl. Environ. Microbiol. 54:878-883.

27. Williams, A. G., and S. E. Withers. 1992. Induction of xylan degrading enzymes in Butyrivibrio fibrisolvens. Curr. Microbiol. 25:297-303. 